\section{Electronic arts imitate life}

\section{Andreas G. Andreou}

THE silicon neuron described by $M$. Mahowald and R. Douglas on page 515 of this issue' is a 'neuromime' which they have built using state-of-the-art microchip techniques, a silicon circuit that mimics the electrical behaviour of real nerve cells. To unearth an appropriate quote from an editorial ${ }^{2}$ by John W. Moore over 30 years ago, the authors report on "an interesting, informative and perhaps provocative example of the wedding of electronic arts and concepts" to one of the life sciences.

\section{Realistic model}

Mahowald and Douglas are scientists who have mastered the art of designing analog microchips using very-large-scaleintegrated (VLSI) circuit technology, which they combine with neurophysiological principles to implement a realistic model of cortical neurons. This is the same technology ${ }^{3}$ that is used to build the processor in the personal computer and the microcontrollers that fine-tune the performance of automotive engines. But whereas the latter systems use digital signals (which have only two states, the on state and the off state) and operate over discrete time intervals, analog ${ }^{4}$ VLSI silicon devices use signals that are graded and continuous in time. The power of VLSI technology lies in its ability to realize complex information processing in a very small volume, with minimal power dissipation and at low cost.

The silicon neuron is an attempt to harness the power of analog VLSI technology in a novel and exciting way to mimic the function of neurons and neural networks. The reported model of the neuron employs four active channels that have simplified dynamics. Real active channels are ion-carrying pores in the cell membrane that are instrumental in the electrical function of the cell. The silicon counterpart devised by Mahowald and Douglas uses a small number of electronic components native to the underlying technology, operates in continuous time and consumes minute amounts of energy. Thus many silicon neurons can be integrated and interconnected on a piece of silicon no larger than a thumbnail.

Several stereotypical neuronal properties are faithfully replicated by the silicon neuromime. When it is excited by a constant electrical current at the place that corresponds to the nerve cell body, the interactions between the active channel currents result in spiking behaviour reminiscent of action potentials recorded from living cells. The neuromime also shows adaptation, an important property of biological neurons. When stimulated, a neuron initially fires a rapid burst of action potentials, but after a while the firing rate tails off. This is why a loud noise is annoying at first but less so with time.

The obvious application of these neuron models is in improving our understanding of brain function ${ }^{5}$. This is a synthetic approach to modelling the nervous system which until now has been studied using software simulations running on massively parallel digital supercomputers. For example, simulation results have been reported recently for dynamic models of the horizontal cell network $^{6}$ in the retina, a part of the brain that is very well studied. These are simulations of realistic Hodgkin-Huxley neuron models, much like the silicon neuromime reported by Mahowald and Douglas, each having seven different ionic channels with parameters obtained from neurophysiological studies.

Simulations of a few hundred thousand horizontal nerve cells take a few minutes of supercomputer time when the stimulus has a duration of a few seconds. Similar simulations take half a day on a state-of-the-art engineering workstation. It should be noted though that horizontal neurons do not spike and that their response is analog. Networks of higher complexity, with feedback connections and a larger number of spiking neurons, could be troublesome because they are described by nonlinear differential equations that are stiff - that is, they require extremely small integration steps and a prohibitively large number of iterations.

On the other hand, an analog VLSI model, although not as accurate as digital simulations, uses the physics of the circuit elements to capture the dynamics of the problem. Therefore, we simply power up the circuit and observe the changes of voltages and currents to obtain the simulation results. This physical model has noise, irregularities and nonlinearities like any real system, including the neurons in the nervous system - properties that are computationally expensive to simulate on a digital computer. So analog VLSI could emerge as the technology of choice for modelling the nervous system. Largescale analog simulation need not be restricted to biological systems, but can be extended to modelling systems in physics and other life sciences.

Engineers and computer scientists are working in the opposite direction, towards building a new generation of sys- tems with brain-like capabilities ${ }^{7}$. They have the same options as the neuroscientists: software implementations on digital computers and novel hardware. For example, work in computational vision has shown that parallel analog algorithms can solve computationally demanding early-vision problems ${ }^{8}$. Compact integration of these analog algorithms in hardware requires circuit designs that are efficient in area and consume a manageable amount of energy. In this micropower regime of operation, a new set of problems arises: devices are very slow, their characteristics are nonlinear and strongly temperature dependent. Furthermore, as we put more and more devices on a chip our computational primitives become less precise. Finally, these systems should be provided with appropriate input transducers to convert the actual physical stimuli of the real world into electrical signals.

\section{Making progress}

There are still several challenges. How can analog systems achieve the dynamic range that a particular problem requires? What should the signal representation be? How can one achieve the precision that a problem requires with inherently imprecise components? How does one guarantee that the results are independent of temperature? How can the transduction of optical, mechanical and chemical signals for vision, audition and olfaction be handled? By ingenious use of the silicon-device physics, innovative circuit-design principles and careful interpretation of the organizing principles in neural sytems, researchers are making progress in these areas ${ }^{9}$.

From the point of view of computer science, concerns such as those outlined above are a reminder that computation is a physical process governed by the physics of the computational substrate. A science of computation is emerging from both biological and engineering concerns that someday may lead to large-scale information-processing systems that approach the efficiency and robustness of neural systems.

Andreas G. Andreou is in the Department of Electrical and Computer Engineering, The Johns Hopkins University, Baltimore, Maryland 21216, USA.

\footnotetext{
Mahowald, M \& Douglas, R. Nature 354, 515-518 (1991).

2. Moore, J. W. Proc IRE Spec. ISsue on Biomedical Electronics 47 (November 1959).

3. Conway, L. \& Mead, C. Introduction to VLSI systems (Addison-Wesley, Reading, Massachusetts, 1980).

4. Mead, C. A. Analog VLSI and Neural Systems (AddisonWesley, Reading, Massachusetts, 1989).

5. Sejnowski, T. et al. Science 241, 1299-1305 (1985)

6. Winsiow, R. \& Knapp. A. G. Prog. Biophys. molec. Biol. 56. $107-133$ (1991).
Winsiow, R. \& Knapp.

7. Hopfield. J. Proc. natn. Acad. Sci. U.S.A. 79. 2554-2558 (1982).

8oggio, T, et al. Nature 317, 314-319 (1985)

9. Sánchez-Sinencio, E. (ed.) IEEE Trans. on Neural Net Spec. Iss. on Hardware Implement. 2 (March 1991).
} 